\title{
Application of generalized logistic equation for b-value analysis in fracture of plain concrete beams under flexure
}

\author{
Nitin B. Burud, J.M. Chandra Kishen* \\ Dept. of Civil Engineering, Indian Institute of Science, Bangalore, India
}

\section{A R T I C L E I N F O}

\section{Keywords:}

Acoustic emission

Plain concrete

Damage

b-value

Generalized logistic equation

Structural health monitoring

\begin{abstract}
A B S T R A C T
This work investigates the effect of finite size and evolution of fracture process zone on b-value of acoustic emission in concrete beams. It is shown that the $\mathrm{AE}$ b-value determined using the Gutenberg-Richter (GR) law varies with beam size and invalidates universality. Since GR law fits the frequency-magnitude curve partially, a recently proposed Maslov's generalized logistic equation (GLE) is used for b-value analysis. The existence of cut-off magnitude and nonlinearity of frequency-magnitude distribution is explained through crack interaction mechanisms occurring within the FPZ. The growth of micro and macro cracking in concrete is observed to follow logistic or sigmoid growth law rather than the power law. The b-values obtained from GR and GLE are compared and it is found that the b-value of GLE correlates well with the effective crack length during damage process of plain concrete thereby exhibiting damage compliant behavior which could be used in health monitoring of structures.
\end{abstract}

\section{Introduction}

Gutenberg-Richter (GR) law [1] has ruled the earthquake statistics for decades after its inception and still continues to do so, not only in seismology but in many other fields too. With the ease in application, it represents a nonlinear chaotic system to appear somewhat linear and systematic. In fact, at any given time instant, it provides a snapshot of a dynamical system in the form of an ogive. The GR law is regarded as a power law, characterizing the frequency-magnitude relation of earthquake events and the exponent of this power law is known as b-value. The existence of power law entails self similarity and scaling in the emerging processes like earthquake. Furthermore, $\mathrm{b}$-value tends to a constant value $(b \approx 1)$ near criticality exhibiting universality. Consequently, GR law becomes more profound and generic for applications due to its manifestation of universality and self similarity.

Universality establishes a link between different complex processes participating in an emerging system to an universal scenario (yet unknown) at critical transformations. Self similarity allows an extrapolation to predict the crucial large magnitude events from smaller ones. Although, universality and self similarity are relevant properties, many researchers have observed deviation in the cumulative frequency distribution (CFD) contradicting pure GR law. The oversimplification of CFD by GR law does not account for small magnitude events and it also disregards large magnitude cut-off. The cut-off magnitude and log-concavity are important features of CFD and should not be neglected but rather accounted for in the b-value analysis. In the present work, the log-concavity along with cut-off magnitude of CFD are addressed together as nonlinearity of CFD in a general sense signifying nonlinear shape on both semi-log and linear scales.

As GR law was first introduced for statistical analysis of earthquakes and applied without any modification to acoustic emission

\footnotetext{
* Corresponding author.

E-mail address: chandrak@iisc.ac.in (J.M. Chandra Kishen).
} 


\section{Nomenclature}

$a_{0} \quad$ notch depth

$a_{e} \quad$ effective crack length

$b_{G R} \quad$ b-value using GR law

$b_{G L E} \quad$ b-value using GLE

C constant in GLE

D overall beam depth

$K_{I} \quad$ mode-I stress intensity factor

$L \quad$ beam span

$m \quad$ magnitude of events $\begin{array}{ll}m_{u} & \text { upper cut-off magnitude limit } \\ m_{m}^{N} & \text { cut-off magnitude at N-th event }\end{array}$

$N$ number of events

$N_{\max } \quad$ maximum number of events at failure of beam

$s \quad$ rate parameter of GLE

$t_{0} \quad$ time of 100th event (considered as initial time)

$t_{i} \quad$ time of i'th event

$\alpha \quad$ shape parameter of GLE

$\Delta m \quad$ magnitude binning interval

$\Delta t \quad$ time binning interval

$\sigma \quad$ stress

due to its reasonable interpretation of this phenomenon [2], it is necessary to review its development in seismological studies. Therefore, the present work mostly reviews seismological and geophysical literature. Mainly, three different types of approaches are followed in literature to improve b-value estimation: (1) employing statistics based formulation by accounting nonlinearity while preserving pure GR law, (2) modifying GR law at large magnitudes by associating it with a polynomial or exponential or logarithmic cut-off and (3) suggesting an alternative model or generalizing GR law to consider the shape of distribution. By idealizing the occurrence of earthquakes as a Poisson process, the first approach with validity of GR law has yielded various statistical b-value estimates such as maximum likelihood estimate or Aki's formula [3], improved b-value [4], Weichert's formula [5], generalized AkiUtsu $\beta$-estimator [6] and few others. An alternate approach based on robust fitting [7] has been proposed which is shown to be more efficient than maximum likelihood estimate. Cut-off on maximum magnitude approach has resulted in many of upper bound models such as tapered GR, truncated Gamma law, truncated GR, etc. The third approach has not received significant contribution except for a few which are noted further. The recent development in Tsallis nonextensive statistical mechanics has offered a generalized form of GR law [8-10] based on Tsallis entropy. A Bayesian approach [11] is also notable in the category of generalized GR law. Apart from the above mentioned contributions, an approach based on logistic equation proposed by Maslov et al. [12] is distinct due to its fractional power-law exponent. This two parameter model regards nonlinear CFD over whole magnitude range with soft cut-off for larger magnitudes. The overall shape of CFD suggest that the growth of microcracks is logistic or sigmoid rather power law growth. The b-value obtained from logistic equation exhibits damage compliant behavior as concluded in the present work. Therefore, the present work details application of generalized logistic equation for b-value analysis of acoustic emission in single edge notched (SEN) plain concrete beams.

Acoustic emission phenomena has a close resemblance to earthquakes [13] despite voluminous contrast on the temporal, spectral, spatial and energetic scales [14]. The acoustic emission (AE) is a non-destructive technique and different features of it have been used for structural health monitoring (SHM) [15-20]. It is an acoustic based passive technique relying on stress waves generated by cracking of material. Development of AE has been greatly influenced by seismological studies and nonetheless, AE has contributed in understanding the complex earthquake process through laboratory experiments on rocks. The ubiquitous GR law has been applied to $\mathrm{AE}$ and determination of b-value has become an essential part in most of the AE studies.

Application of GR law for b-value analysis is usually carried out to assess the progress of damage in a structural element. Convergence of b-value approximately towards unity is considered as a sign of near failure. Qualitative drop of b-value with increasing stress is consistent throughout the damage progress but quantitatively it is still uncertain to associate b-value to a specific damage level. Furthermore, according to fracture mechanics, the crack size depends not only on stress but also on stress intensity at crack tip as the crack propagates. Therefore, if b-value is only correlated to stress then the conjecture of micro and macro crack distribution associated with b-value is also ambiguous (same b-value does not imply same crack size distribution). Most importantly, GR distribution fits only to the tail portion of CFD neglecting small magnitude events, thereby making the b-value as a biased indicator. On the other hand, the prominent size effect in concrete has been attributed to varying fracture process zone size. Thus, the increasing width of fracture process zone with size indicates widely distributed microcracks around the main crack. Therefore, large size beams should result in higher b-value due to relatively higher microcracking compared to small size beams. Such effect of finite size on b-value has not yet been discussed in the literature. In this work, the nonlinearity of CFD is dealt by employing Maslov's generalized logistic equation (GLE) with two parameters.

The present work is organized as follows. Section 2 introduces Gutenberg-Richter law followed by a brief discussion on the universality of b-value. Maslov's generalized logistic equation is introduced in Section 3. The b-value is a parameter which represents FPZ in the statistical domain and hence fracture process in concrete and evolution of FPZ is described in Section 4. Experimental details with analysis techniques used in the present work are given in Section 5 . The test results of geometrically similar beams that are tested are described in Section 6. The details of fracture process zone as an interactive phenomenon of micro and macro crack is thoroughly discussed with experimental observation in Section 7.1 and the nonlinearity of CFD and existence of cut-off magnitude is justified. Application of GLE to AE data acquired from tested beams is demonstrated in Section 7.3. The damage compliance behavior of b-value obtained from GLE is shown in Section 7.4. The conclusions arising from this study are briefed in Section 8.

\section{Gutenberg-Richter Law and the universality of b-value}

The ubiquity of Gutenberg-Richter (GR) law in many natural phenomena has been studied and confirmed. Although it was 
fundamentally proposed for earthquake occurrences in seismology, it has been adopted and applied in many other areas too. Despite ubiquity, GR law has received criticism due to its broad generality which makes it difficult to validate in many other situations. In fact, in seismology itself it holds true only for the finite range of magnitudes [21,22]. The GR law can be expressed as follows,

$$
\log _{10}(N \geqslant m)=a-b m
$$

where $\mathrm{N}$ is the number of events greater than magnitude $m, a$ is referred as productivity and $b$ is the negative slope of CFD over magnitude plotted on log-linear scale.

The GR law has been applied extensively for b-value analysis of AE events. However, GR law partially represents the CFD of AE. There are possible reasons for it being partially applicable to AE data, one of which is the incompleteness of small magnitude data as a consequence of thresholding used during its acquisition. Further, saturation of large magnitude events results in maximum amplitude cut-off, which the GR law fails to consider. Nevertheless, the CFD of AE is not linear and requires another model which can effectively describe it. The universality of b-value is a conviction followed by AE community borrowed from seismology. In seismology, the bvalue is used to predict the large (largest) size earthquake by extrapolating smaller earthquakes for which existence of universality can be considered useful. On the other hand, AE in structural health monitoring (SHM) is required to estimate damage progress and not to extrapolate large size events and therefore small variations in shape of CFD should also be regarded. This basic difference in application of b-value in both seismology and SHM is important and needs to be emphasized. The universality of b-value is a topic of debate for several years and many studies have supported its existence [23,24]. The present work provides evidence for non-existence of universality by an experimental study using acoustic emission.

The universality of b-value has been a conundrum for decades and its observed variability has added further dilemma among researchers. As a measure of variation in power law distribution, the b-value obviously depends on the underlying physics of the system. Therefore, the universality of b-value indicates that different emerging systems have the same universal physical phenomenon behind them. Whether universality exists or not, several factors affecting b-value have been recorded and studied by many seismologists [25]. Such studies are found to be scarce in the literature dealing with AE of concrete. Factors affecting b-value in concrete fracture include heterogeneity [26,27], stress level [4,28-30], shape of the tail distribution affected by cut-off magnitude [31,32], different methods of b-value evaluation [3,4], sample size [33], magnitude binning [34,3,35,36] and most important of all is the specimen size. These factors can be broadly distinguished as numerical, material and geometry dependent. The b-value dependence on the numerical factor can be reduced by selecting appropriate sample size and binning magnitude. The material and geometry dependence cannot be avoided but can be quantified. Although, the major problem with b-value analysis is more elemental, it needs to be addressed based on the physics of the problem. This elemental problem is nothing but the validity of GR law itself. Therefore, the present work advocates use of generalized logistic equation for b-value evaluation instead of GR law. Besides application of GLE, the effect of finite size is also investigated by testing geometrically similar concrete beams of three different sizes under flexure.

\section{Generalized logistic equation}

Recently, Maslov et al. [12] proposed a new equation to study the statistics of earthquake distribution by calling it as "the generalized logistic equation". A special case of Maslov's generalized logistic equation can be written in the following form,

$$
P(x)=\frac{C e^{\frac{-s}{1-\alpha} x^{1-\alpha}}}{1+C e^{\frac{-s}{1-\alpha} x^{1-\alpha}}} \text { for } 0 \leqslant x<\infty \text { and } 0 \leqslant \alpha<1
$$

Eq. (2) is not a perfect cumulative distribution function but can be considered approximately as CFD for sufficiently large $C$, where $C$ is a constant and $\alpha$ and $s$ are parameters of function $P(x) . P(x)$ is an approximation to the number of all elements with size greater than $x$ and $x$ is a representative variable of the size of an element in a structure (e.g., the magnitude of earthquakes, the amplitude of acoustic emission events, etc.). $P(x)$ varies from $C /(1+C)$ for $x=0$ to 0 for $x \rightarrow \infty$. For sufficiently large $C(C /(1+C) \approx 1)$, the denominator of Eq. (2) is a normalizing function (similar to partition function used in statistical mechanics). Therefore, in order to arrive at an analogous GR law expression, removing the denominator and replacing variable $x$ by magnitude $m$ results in,

$$
N(m)=C e^{\frac{-s}{1-\alpha}} m^{1-\alpha}
$$

Eq. (3) can also be obtained as a first-order approximation of Eq. (2) by power expansion. Eq. (3) can be written in a logarithmic form as,

$$
\log _{10} N(m) \approx \log _{10} C-\frac{s}{1-\alpha} m^{1-\alpha} \log _{10} e
$$

Eq. (4) is analogous to the Gutenberg Richter law shown by Eq. (1) for $\alpha=0, a=\log _{10} C$ and $s=b$. The above equation considers the nonlinear relation between magnitude $m$ and cumulative event number $N(m)$ contrary to linear relation assumed in GR law. The role of $\alpha$ can also be thought of as a penalty on GR law due to nonlinear relation between $N(m)$ and $m$. Therefore, the new b-value $\left(b_{G L E}\right)$ based on approximation of generalized logistic equation and equivalent to the GR law can be written as,

$$
b_{G L E}=\frac{s}{1-\alpha} \log _{10} e
$$

As Eq. (4) is simply a first order approximation of Eq. (2), for b-value analysis, the estimation of parameters $s$ and $\alpha$ should be 
performed using Eq. (2) itself. Fig. 1 shows the behavior of Eq. (2) with respect to varying parameters $\alpha$ and $s$ on linear and log-linear scales for comparison. The variation of $\alpha$ is plotted in Fig. 1a for a constant value of $s=3.5$. The head and tail portion of the curves are dominated by $\alpha$ which ensures smooth transition from small to large magnitudes. It is evident that larger $\alpha$ accounts for fewer small magnitude events while small $\alpha$ accounts for the relatively large number of small magnitude events. Fig. $1 \mathrm{~b}$ and $\mathrm{c}$ are plotted for two different values of $\alpha(\alpha=0, \alpha=0.5)$ against varying s-values. For $s=1$ in Fig. 1b, the slope of the curve on the log-linear scale is zero indicating an uniform distribution of events of all magnitude. Increasing s-value reduces the contribution of large magnitude events in CFD therefore the slope of the curve is dominated by s-value. It follows that the s-value is a rate parameter and $\alpha$ is a shape parameter of GLE model. Consequently, $\alpha$ is the parameter which accounts for nonlinearity and cut-off magnitude of CFD. The GLE

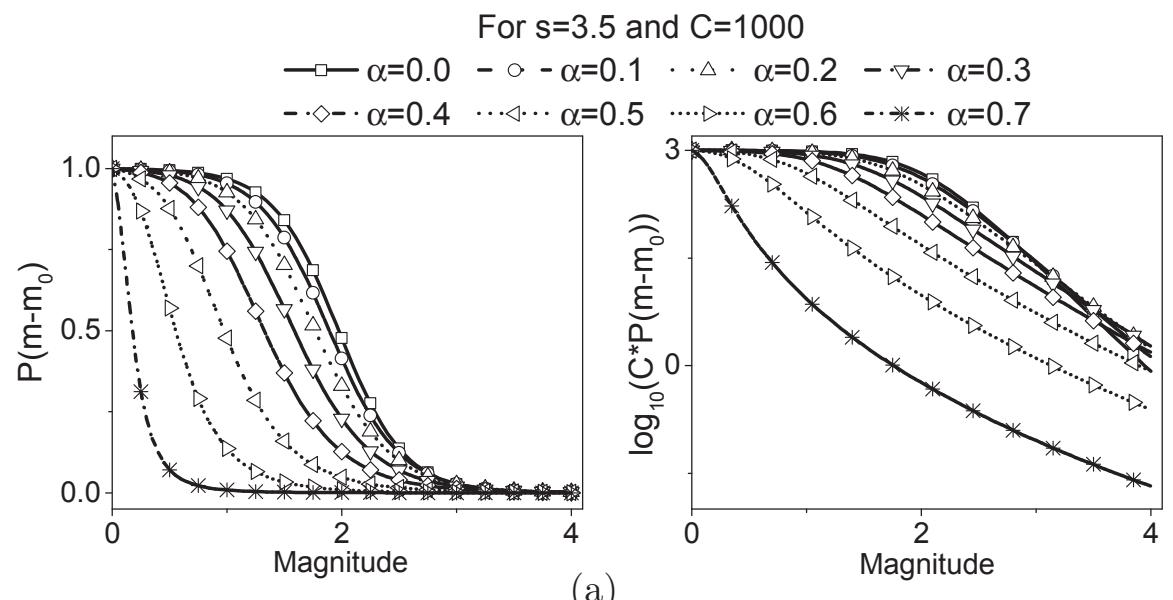

(a)

For $\alpha=0$ and $C=1000$

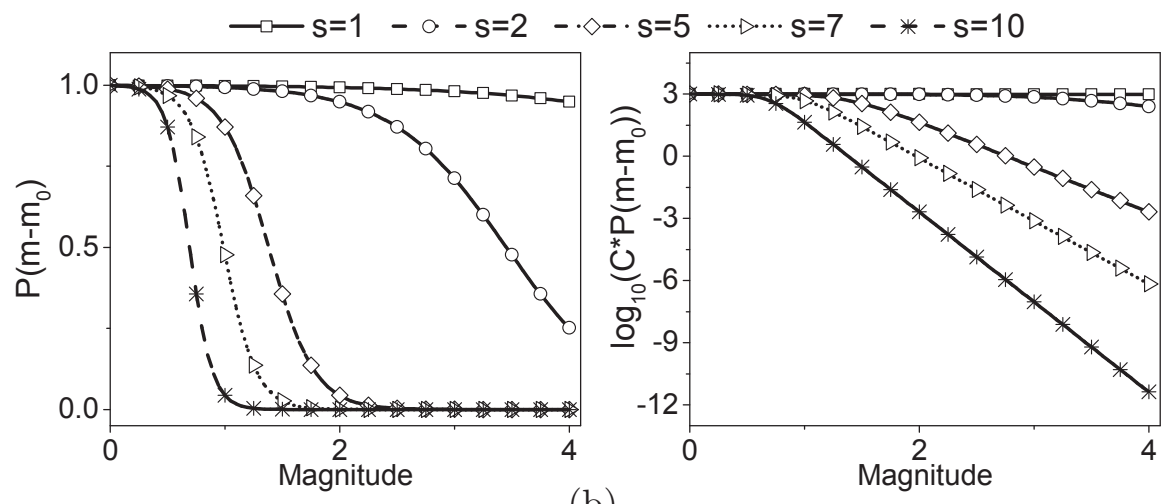

(b)

For $\alpha=0.5$ and $\mathrm{C}=1000$

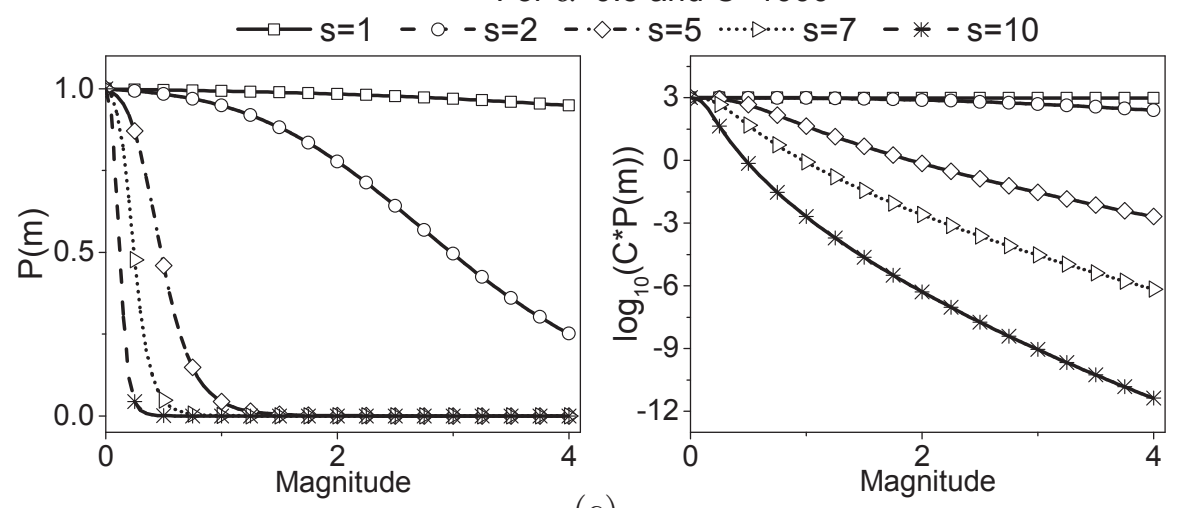

(c)

Fig. 1. Plot of Eq. (2), (a) for $C=1000, s=3.5$, varying $\alpha=0-0.7$, (b) for $C=1000, \alpha=0$, varying $s=1-10,(c)$ for $C=1000, \alpha=0.5$, varying $\mathrm{s}=1-10$. 
model sets a soft limit over large magnitude events, analogous to Gamma law advocated by Kagan [37,38]. Soft magnitude cut-off considers the possibility of large magnitude events with progressively smaller probabilities compared to GR law and therefore it allows smooth transition of the dissipative dynamic system.

As discussed above, GLE appears to be an appropriate candidate for b-value analysis. However, before moving to b-value evaluation using GLE, it is necessary to briefly introduce fracturing process and fracture process zone of quasi-brittle material such as concrete.

\section{Evolution of fracture process zone in quasi-brittle materials}

Although resemblance between occurrence of earthquakes and AE events does exists, the mechanics behind both of these phenomena have a vast difference. The earthquakes originate from fault lines due to sliding movement of tectonic plates. On the other hand, the development of FPZ in quasi-brittle material, like concrete, originates from cracking of material due to local tensile stresses. The area of fault plane is considered as an invariable, in seismology. Whereas, the volume of fracture evolves in size during fracture process of concrete. In fact, volume of the FPZ depends on structure size, geometry and loading conditions to which the material is subjected. Therefore, it is necessary to understand the evolution of fracture process in concrete to appreciate b-value variation in AE. Fracture process zone is a cluster of micro and macroscopic cracks around the crack tip produced by stress redistribution and energy dissipation mechanism due to underlying heterogeneity as shown in Fig. 2. In quasi-brittle materials like concrete, the heterogeneity primarily arises due to its constituents of varying particle sizes from fine to coarse aggregates and it is further elevated by discontinuities and pores developed during the hydration of cement. The randomly dispersed discontinuities and pores play the role of probable sites for crack initiation due to stress concentration. At the crack initiation stage, microcracks develop and coalesce due to applied stress and form a macrocrack near the crack tip oriented perpendicular to the principal tensile stress direction. After crack initiation, the fracture mechanism becomes an interactive process between various crack sizes [39]. Microcracks usually occur around the main crack and their density reduces as the distance from the crack tip increases. Otsuka et al. [40] studied the development of FPZ in concrete under tensile loading using AE with X-ray and proposed the existence of a sub-zone within the FPZ and named fracture core zone (FCZ) as shown in Fig. 2. Similar behavior by considering AE energy is recorded in [41]. FCZ is an area of densely distributed microcracks which further develops into a main crack. Such dispersed microcracking around a macrocrack offers a bi-fold contribution to fracture process by relieving the stress singularity as a consequence of stress redistribution which is accompanied by energy dissipation. This bi-fold contribution adds shielding effect at the main crack tip often documented as toughening mechanism by microcracks. Not all, but the majority of microcracks participate in shielding while others amplify stress intensity at the main crack tip. Microcracks which are far away from the FCZ do not interact significantly with the main crack and remain idle after dissipation of energy. Therefore, shielding is a collective mechanism of interaction between micro-micro and macromicro cracks. The interactive process of different crack sizes is further elaborated with experimental observations in Section 7.

\section{Experimental program}

\subsection{Experimental setup}

An experimental program is designed to study the b-value behavior of geometrically similar plain concrete beams of three different sizes. The geometrically similar notched plain concrete beams are casted from the same concrete mix. The mix design of concrete is done using the ACI method and the mix proportion of the cement, fine aggregate and coarse aggregate obtained is 1:1.86:2.61 by weight. The maximum size of coarse aggregate is $12 \mathrm{~mm}$ and the fine aggregates (river sand) pass through $4.75 \mathrm{~mm}$

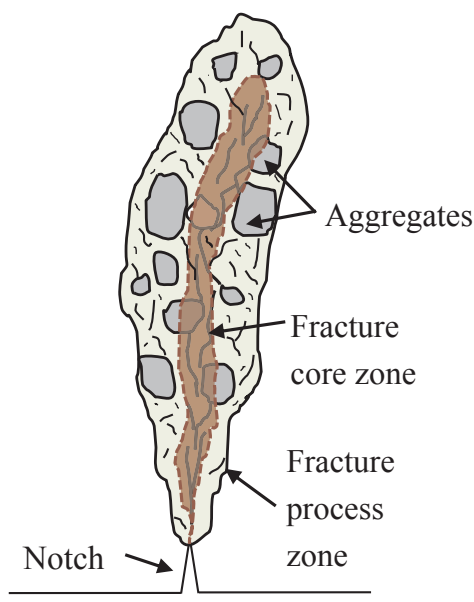

Fig. 2. Schematic diagram of FPZ and FCZ. FCZ is shown in red shaded region. (For interpretation of the references to color in this figure legend, the reader is referred to the web version of this article.) 
sieve. A water to cement ratio of 0.5 is used for preparing the concrete mix. Table 1 gives the geometrical details of the beams. A computer controlled servo hydraulic machine is employed for testing the beams in flexure under three-point loading using crack mouth opening displacement (CMOD) control. Monotonic loading rate is set to $1 \mu \mathrm{m} / \mathrm{s}$ for all specimens. Midpoint deflection of beams is measured using a linear variable differential transformer (LVDT), while the load is recorded using a load cell of $35 \mathrm{kN}$ capacity. Three specimens are tested for each size of beam.

A Physical Acoustic Corporation (PAC) system is used to monitor the acoustic emission throughout the test. Six resonant type R6D AE sensors are mounted on beams as shown in Figs. 3 and 4. R6D sensors having sensitivity and frequency response over the range of $35-100 \mathrm{kHz}$ with resonant frequency around $55 \mathrm{kHz}$ are used. AE sensors are attached to concrete surface using vacuum grease as a couplant and adhesive tape is used to ensure fixity of sensor to the surface. Due to weak strength of AE signals, preamplifier with $40 \mathrm{~dB}$ gain was set for signal amplification. A threshold limit of $40 \mathrm{~dB}$ was set for background noise reduction. Sampling rate of $1 \mathrm{MHz}$ was used to ensure good time and signal frequency resolution. Signals below the threshold level are neglected. In order to obtain the crack length as a function of loading, digital image correlation (DIC) technique is adopted. The surface of the beam specimen is sprayed with speckles of a black paint and images of the beam are captured continuously during the loading stages by a digital camera mounted on a tripod.

\subsection{Analysis of AE data}

Continuous monitoring of the beams up to failure by using AE and DIC techniques resulted in a large data generation which are analyzed. AE can be thought of as a passive acoustical microscope which provides a glimpse of the internal cracking mechanism of the material. On the other hand, a whole-field based DIC technique provides surface information regarding deformation/strain development and cracking due to the applied loading. According to the AE terminology, AE signal acquired by a single sensor is called as a "hit" and if the same hit is acquired by multiple sensors then it is counted as an "event". Events can be localized in space by using triangulation method based on differences in arrival time of the stress waves. An event is considered to be originated from a single micro or macro crack source, though the possibility of multiple crack occurrence cannot be denied. The amplitude of AE signal is considered to be correlated to the microcrack volume and therefore often used as an alternative to AE energy. In the present work, the amplitude of hit acquired by the first sensor, in a group of sensors which acquired the event, is considered as the source amplitude. The magnitude scale is derived by subtracting threshold amplitude of $40 \mathrm{~dB}$ from the acquired raw source amplitudes of stress waves ranging from $40 \mathrm{~dB}$ to $99 \mathrm{~dB}$ and then dividing it by 20 makes it useful for b-value analysis. The factor 20 accounts for conversion from voltage to decibel scale with reference voltage of $1 \mu \mathrm{V}$ referred to the preamplifier input. For example, $65 \mathrm{~dB}$ amplitude will be denoted as $1.25((65-40) / 20)$ on magnitude scale. The recorded magnitude for each event are further used for the b-value analysis. The referred meaning of magnitude in $\mathrm{AE}$ is straight forward and should not be confused with different magnitude scales used in seismology.

There are two methods for windowing on time series for the b-value analysis usually followed in the literature: (i) instantaneous b-value by considering a fixed length of time/event sliding window and, (ii) long-term b-value by considering all events occurred before a particular time instant. The long-term b-value is preferred as it represents overall b-value variation throughout the test duration. If $t_{0}$ is the time of test initiation, then at any time $t_{i}$ all the events occurred during interval $t_{0}$ to $t_{i}$ should be used to evaluate the long-term b-value at time $t_{i}$. The time $t_{0}$ is considered as the time of occurrence of 100th event and therefore at any time instance $t_{i}$, the number of events is always greater than 100 due to the cumulative effect. Increment of 100 events is considered such that $t_{i}=100 . i+100$, for $i=0,1 \ldots$. Magnitude binning also plays an important role in the accuracy of b-value and therefore the magnitude interval is kept as low as possible i.e. $\Delta m=0.05$ (magnitude binning interval is $1 \mathrm{~dB}$ with respect to source amplitudes). Eq. (2) is then fitted to CFD using particle swarm optimization and parameters $s_{i}$ and $\alpha_{i}$ are determined for incremental $t_{i}$. Use of particle swarm optimization is the authors personal preference although any other optimization technique can be used as an alternative for parameter estimation. The obtained parameters are then substituted into Eq. (5) to determine $b_{G L E}$. Transformation from linear to loglinear scale is not required for evaluation of $b_{G L E}$ as Eq. (2) implicitly incorporates CFD nonlinearity through exponent $\alpha$. Therefore, GLE discards the CFD linearity pretext which is often emphasized on log-linear scale.

DIC analysis is performed on images captured by high resolution camera using GOM correlate software package [42]. Successive images are captured at random intervals during load application. The crack tip determination is achieved manually by varying strain and displacement threshold on experience basis.

\section{Results of mechanical testing and acoustic emission}

The load versus crack mouth opening displacement (CMOD) response for all the three sizes of beams tested is shown in Fig. 5a.

Table 1

Details of beam dimensions.

\begin{tabular}{llll}
\hline Designation & Depth $(\mathrm{mm})$ & Width $(\mathrm{mm})$ & Span $(\mathrm{mm})$ \\
\hline Small & 75 & 50 & 337.5 \\
Medium & 150 & 50 & 675 \\
Large & 300 & 50 & 1350 \\
\hline
\end{tabular}




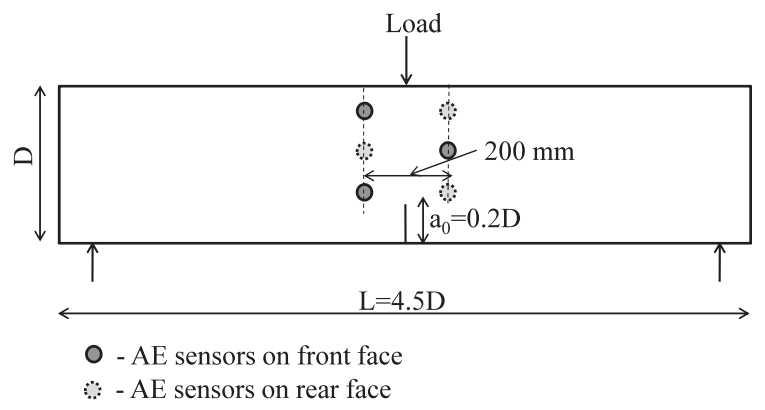

Fig. 3. Beam dimensions with $\mathrm{AE}$ sensor location for large beam.

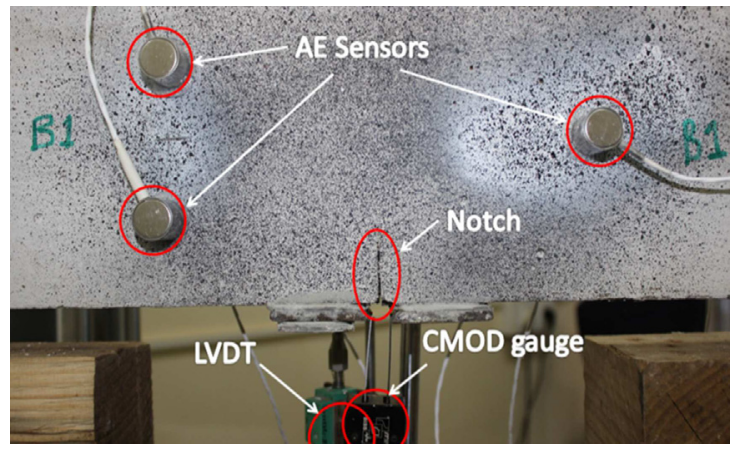

Fig. 4. Beam with AE sensors, LVDT and CMOD gauge.

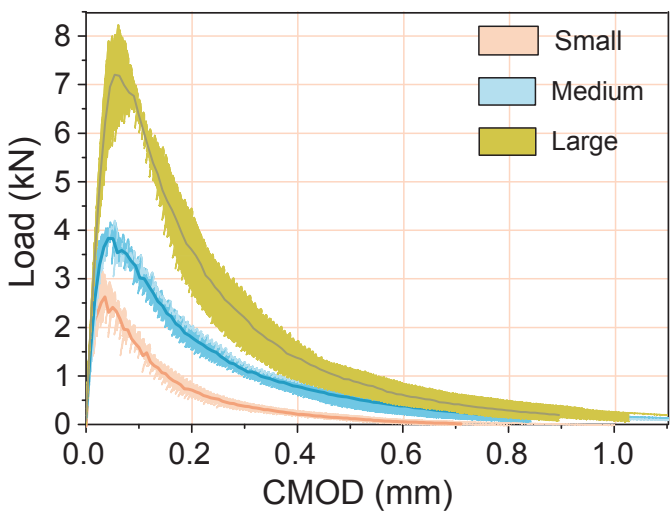

(a)

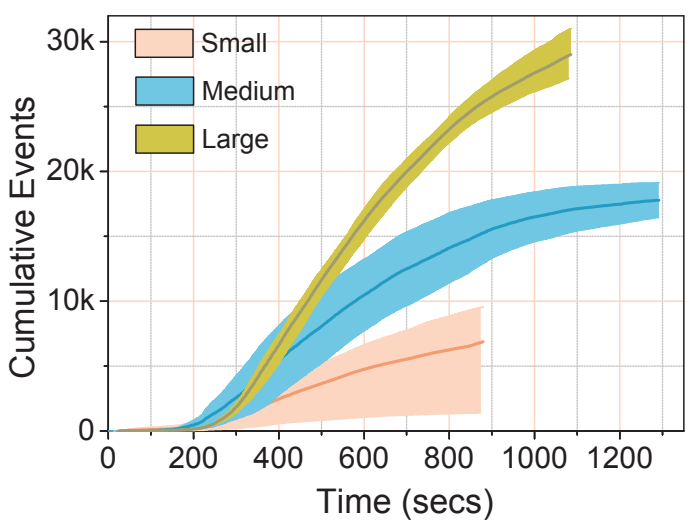

(b)

Fig. 5. (a) Load vs. CMOD and (b) cumulative AE events for three different size specimen (shaded area shows scatter while dark line shows average of the variable plotted).

The shaded region depicts the scatter observed in the response for three specimens of each size. The scatter plot is obtained by filling the area in between curves of each specimen of the same size. The average dark line is obtained by averaging the variable over $\mathrm{x}$ axis. The use of CMOD control allows stable crack propagation resulting in the development of FPZ which is evident from the softening behavior exhibited by the beams in the post-peak regime. Similar scatter is also seen in the acquired cumulative AE events as shown in Fig. 5b. Table 2 lists the observed statistics of AE hits and events during progressive cracking for each specimen. The typical CFD of cumulated event over magnitudes is shown in Fig. 6a at various stages of damage progress. Fig. 6b represents the same CFD on loglinear scale. The GR b-value is usually determined by least square fitting of CFD on log-linear scale. The slope of the fitted line is called as the b-value. Along with nonlinearity, another noticeable feature of CFD is the cut-off magnitude denoted by vertical dotted lines in Fig. 6b. The cut-off magnitude is defined as the highest AE magnitude observed at any instance of damage. The log-linear plot clearly exhibits the incremental cut-off magnitude $\left(m_{\max }\right)$ with the increasing number of events and as an example shown in Fig. $6 \mathrm{~b}$ for $N=100$ by $m_{\max }^{N=100}$. There is also an evidence of upper cut-off limit, irrespective of the beam size at $99 \mathrm{~dB}$ as seen in Fig. $6 \mathrm{~b}$ denoted by $m_{u}$. No event has been observed throughout the experiment above magnitude $m_{u}$ and once this upper limit is crossed, the CFD curve starts shifting upward indicating accumulation of increasing number of events with magnitude $m_{u}$. Such upper limit on 
Table 2

Statistics of AE events and hits.

\begin{tabular}{|c|c|c|c|c|c|c|c|c|}
\hline \multirow[t]{2}{*}{ Size } & \multirow[t]{2}{*}{ Specimen } & \multirow[t]{2}{*}{ Events } & \multicolumn{6}{|c|}{ Hits } \\
\hline & & & $\mathrm{S} 1$ & $\mathrm{~S} 2$ & S3 & S4 & S5 & S6 \\
\hline \multirow[t]{3}{*}{ Small } & B1 & 9008 & 28,938 & 23,766 & 18,254 & 26,175 & - & - \\
\hline & B2 & 1388 & 21,304 & 18,358 & 7491 & 4446 & - & - \\
\hline & B3 & 10,651 & 23,793 & 26,666 & 28,069 & 25,416 & - & - \\
\hline \multirow[t]{3}{*}{ Medium } & B1 & 7956 & 12,386 & 9876 & 12,902 & 8220 & 14,809 & 9744 \\
\hline & B2 & 19,142 & 23,797 & 25,605 & 28,247 & 26,045 & 27,555 & 39,383 \\
\hline & B3 & 16,446 & 26,226 & 24,235 & 25,772 & 18,276 & 26,526 & 12,706 \\
\hline \multirow[t]{3}{*}{ Large } & B1 & 26,664 & 52,979 & 40,254 & 35,464 & 25,037 & 46,583 & 45,987 \\
\hline & B2 & 27,169 & 57,772 & 44,570 & 23,043 & 39,355 & 52,371 & 50,187 \\
\hline & B3 & 34,166 & 54,582 & 46,150 & 40,312 & 48,433 & 58,827 & 52,138 \\
\hline
\end{tabular}

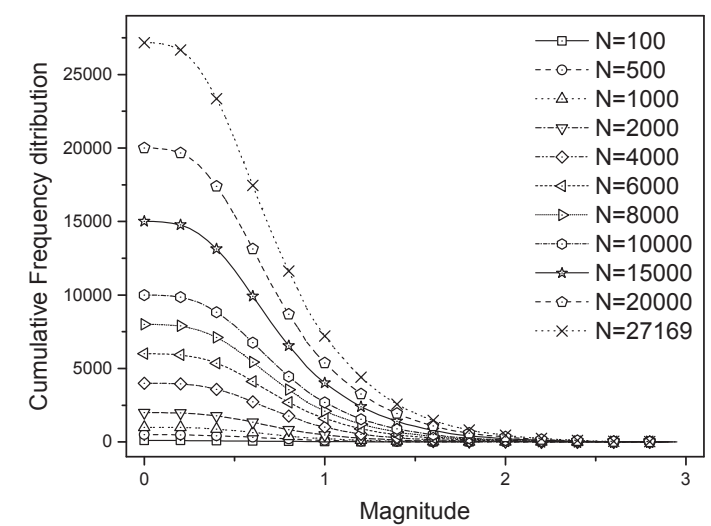

(a)

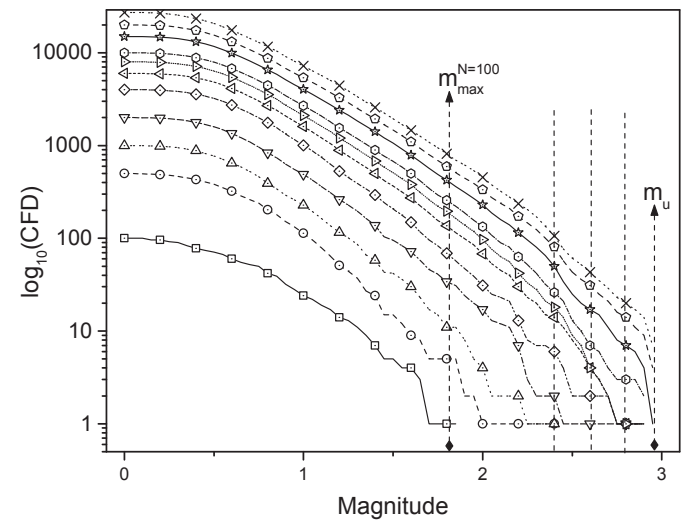

(b)

Fig. 6. Cumulative frequency distribution at different damage instances, (a) linear scale, (b) log-linear scale, vertical dash lines shows cut-off magnitude at different damage instances (refer legend of figure (a) for figure (b) also).

magnitude can be related to material properties at extreme stress conditions. The localized events are shown in Figs. 7 and 8 with data density and color mapped bubble plot showing magnitude of events in decibels. The images analyzed through DIC are shown in Fig. 9 at various stages of loading history. The crack length and crack tortuosity is evident from the analyzed images.

\section{Discussion of results}

\subsection{Effect of finite size and crack interaction on fracture process zone (FPZ) evolution}

The overall topology of the FPZ as shown in Fig. 2 and described in the previous section is well studied and understood in literature. There are two aspects of crack distribution in FPZ which can be observed from AE. The first aspect is the concentration or 


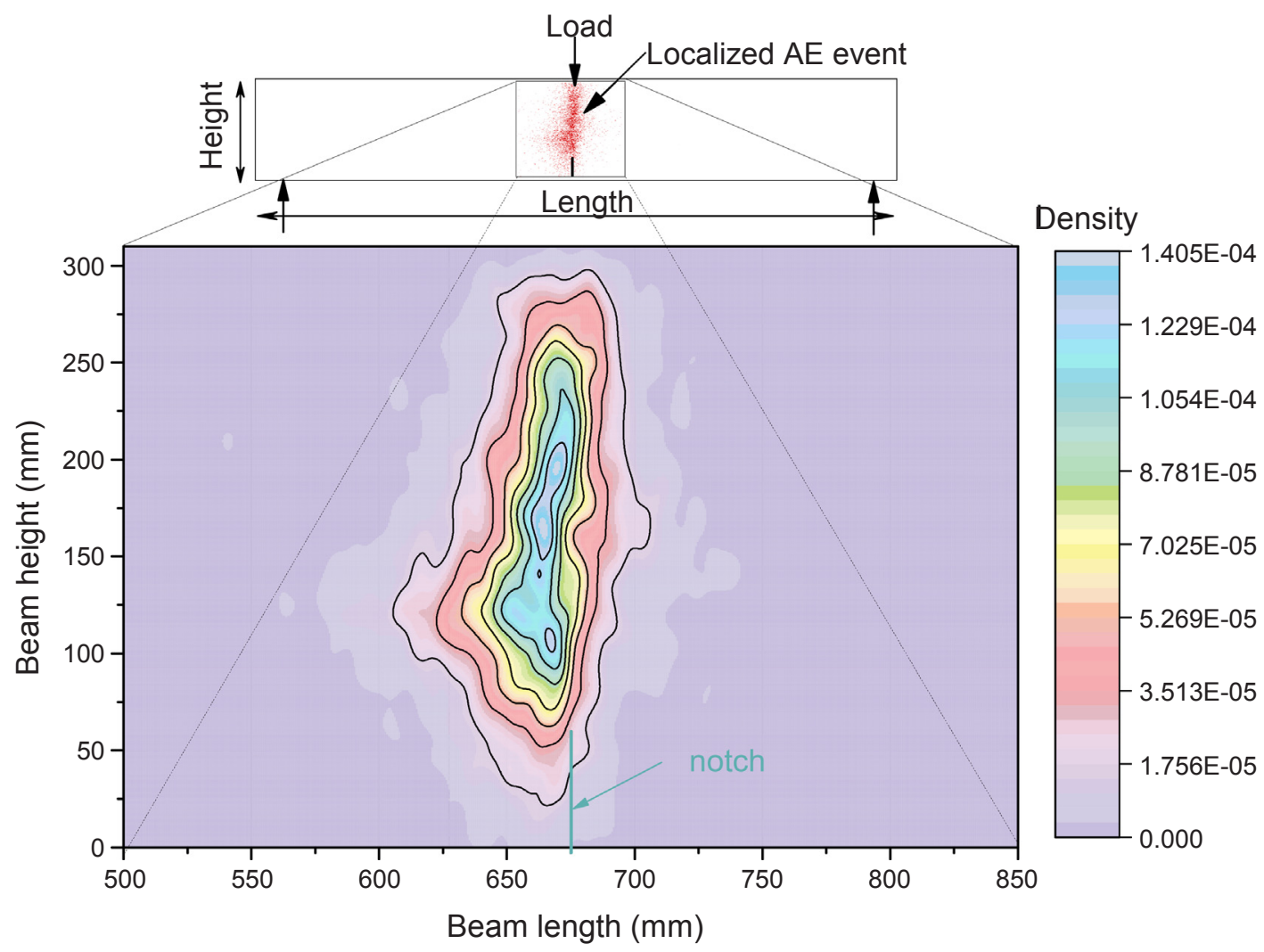

Fig. 7. Qualitative data density of localized events in a large size beam.

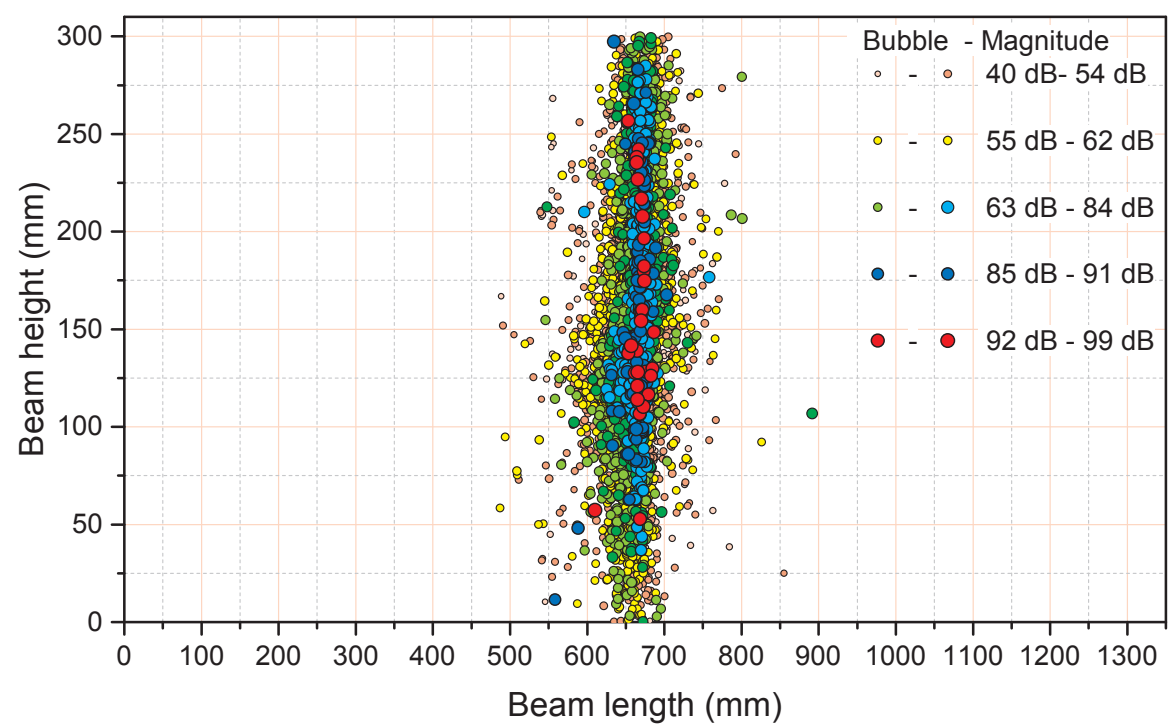

Fig. 8. Localized events in large size beams.

density of the micro and macro cracks with respect to the main crack path. Main crack in single edge notched beams under three point bending initiates at notch tip and progresses towards the loading point. Fig. 7 shows color mapped kernel density contours of localized AE events. The overall shape of FPZ with variation of its width along main crack is evident from Fig. 7. Cracking is highly dense along main crack and reduces farther away from it. The second aspect is the location and number of different sized cracks with respect to main crack location. The bubble plot of AE localized events as shown in Fig. 8 depicts events of different magnitude by color mapped bubbles. It can be clearly seen that large magnitude events are rare and are centrally located. Small magnitude events 


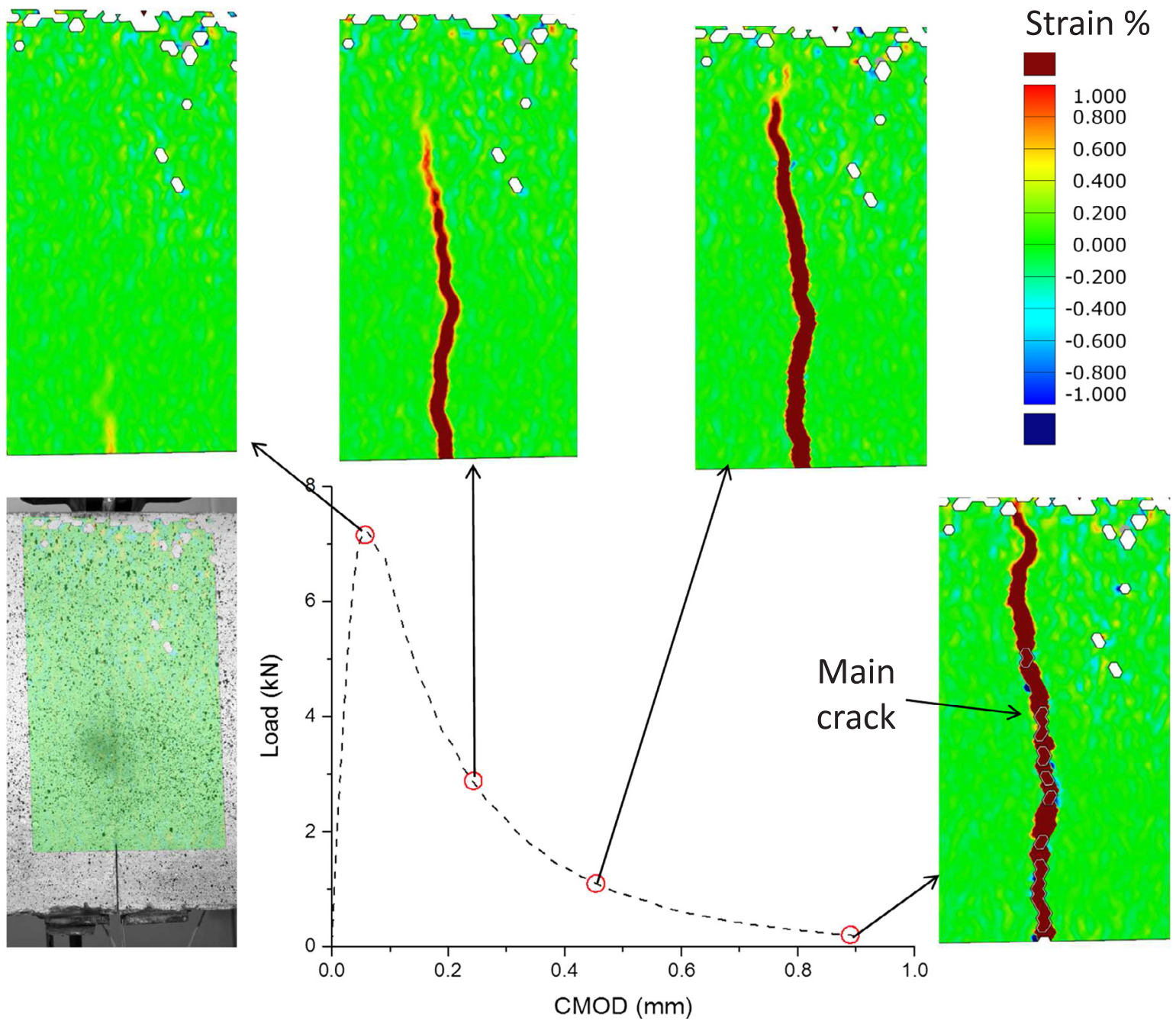

Fig. 9. DIC images at various load level in a large beam.

are dispersed abundantly around large magnitude events. The event locations in Fig. 8 are not in chronological order as the large magnitude events are scattered over small magnitude events to bring out the contrast between various magnitude events. The events between 92 and $99 \mathrm{~dB}$ do not occur frequently but instead occurs in relatively large intervals. Most of these large events have occurred between 100 and $250 \mathrm{~mm}$ depth range in large size beam. There are few large events visible at notch tip around $60 \mathrm{~mm}$ depth due to sudden release of strain energy. In the top range of 250-300 mm, 92-99 dB events are again rare. One can argue that at this range where final failure becomes catastrophic, large size events must be present. However, large size events are less probable near failure due to compressive stresses caused by flexure above the neutral axis and reduced applied load. At this stage, the material is softened and does not have sufficient strength as well as volume to generate large size events.

Although dispersed microcracks around centrally located macrocrack is an overall simple depiction of FPZ, a complex fracture process lies underneath. A macrocrack is more susceptible to growth due to its relatively large size and higher tensile stresses in the mid-span location than the microcracks surrounding it. This implies that, once a macrocrack has emerged, it will grow further and generate a chain of macrocracks of equivalent or bigger size up to failure. This is a typical behavior of brittle materials wherein the chain of macrocracks leads to unstable crack growth heading to catastrophic failure. Contrary to brittle materials, quasi-brittle materials like concrete exhibit stable crack growth due to the toughening mechanism of microcracks as mentioned in Section 4 . Interestingly, macrocracks grow in more restricted environment than the microcracks around it. The first restriction on macrocracks is its interaction with neighboring microcracks during which the energy supplied through loading is consumed, thereby limiting further growth of macrocrack. Secondly, the macrocracks have dimensional restrictions as they grow centrally at the awakening of two new surfaces and therefore their growth is limited in two dimensional space. On the contrary, microcracks are dispersed in volume around the main crack which can grow in three dimensional space. The third restriction arises due to the type of loading and stresses to which the material is subjected. Direct tensile stresses tend to accelerate cracks and compression stabilizes it. The energy dissipation during tensile loading is slow and stable for quasi-brittle material compared to compression where it occurs in large bursts 
close to failure when tested under CMOD or displacement control. During flexural deformation of the beams, the crack growth is stable with the combination of compression and tension. The stresses ahead of crack tip in compression zone restricts further growth of macrocrack. This restriction in the compression zone forces wider dispersion of microcracks which may result in widening of FPZ. Small amounts of cracking might actuate in compression zone too, but these do not lead to any failure in plain concrete beams. Compression zone crack may not help tensile crack propagation directly as both of them are oriented normal to each other. The compression zone cracks soften the material and help tensile cracks indirectly. The softened compression zone might be the reason for observed scarcity of large magnitude events $(92-99 \mathrm{~dB})$ within the top $50 \mathrm{~mm}$ range of the large size beams as shown in Fig. 8 .

Consequently, restricted macrocracks produce incremental magnitude cut-off during progressive damage. Similarly, the size and density of dispersed microcracks diminishes away from the main crack as shown in Figs. 7 and 8 ensuing a flat plateau which is evident in the CFD curve at small magnitudes (Fig. 6). Inevitable thresholding and attenuation also play an important role in acquiring less number of small magnitude events during acoustic emission. In summary, large events experience cut-off while small events are undersized resulting in nonlinear CFD dominated by intermediate size cracks and deviating from the GR law. Gutenberg and Richter also [1] noted such deviation for large earthquake magnitudes which further led to the upper bound power laws [38,43].

The clear distinction between sizes of micro and macro crack has not yet been defined firmly, and it is also experimentally infeasible to associate crack size with AE magnitudes in concrete. Indeed, crack size itself depends on applied stress, stress intensity factor (SIF) and geometry of the specimen. Therefore, a specific crack size at different stages of damage progress will produce different magnitude events depending on SIF and stress condition at that instant. The orientation of the crack also influences the stress wave parameters. Consequently, the AE magnitude distribution progresses as a collective effect of applied stress, SIF, boundary conditions, crack density and their orientation.

\section{2. b-value analysis using GR law}

The conventional b-value analysis using GR law is carried out on the AE magnitudes obtained from beams of different sizes. As beam size increases, eventually to dissipate proportionate amount of applied energy, the volume of FPZ increases. The maximum width of FPZ in a beam depends on maximum aggregate size and uncracked ligament length [40] and therefore for the same maximum aggregate size, the increase in the width of FPZ solely depends on the beam size as shown in Fig. 10. The maximum width of FPZ shown in Fig. 10 is determined as the width of localized AE events in length direction at one standard deviation from the mean ( $\mu \pm \sigma$, where $\mu$ is mean and $\sigma$ is standard deviation, if a data distribution is approximately normal then about $68 \%$ of the data values are within one standard deviation of the mean). Large volume of the FPZ accommodates large number of microcracks due to its larger width and length. The b-value should reflect such relative change in the proportion of micro and macro cracks with size. Consequently, different beam sizes should exhibit different b-values instead of converging to unity. Accordingly, universality of the b-value $(b \approx 1)$ should not hold true for different beam sizes. Such size effect on b-value has not been yet studied and discussed. Fig. 11 shows the b-value determined by least square fitting $\left(b_{L S}\right)$ and Aki's formula $\left(b_{M L}\right)$ at final failure for the three beam sizes, where size dependence of b-value is evident. The $b_{L S}$ and $b_{M L}$ are obtained from GR law and therefore these b-values will be collectively referred as $b_{G R}$ henceforth in the present work. According to Carpinteri et al. [24,44], the energy dissipation in disordered materials takes place in fractal domain with dimension lower than 3, intermediate between surface and volume. Aki [45] related the fractal dimension $D$ to seismic $b_{G R}$ through the relation $D=3 b_{G R} / c$ with $c=1.5$. Similar relation for $b_{G R}$ and fractal dimension based on cumulative number of AE events and characteristic linear dimension has been proposed by Carpinteri et al. [24]. The observed average variation of $b_{G R}$ for the present study is $1.15,1.29$ and 1.33 resulting in fractal dimension of $2.3,2.58$ and 2.66 for small, medium and large size beams respectively. Therefore, an alternate explanation for size effect on $b_{G R}$ based on fractal theory could be that the damage localization in flexure of concrete beams is shifting from two dimensional to three dimensional fractal space with increase in the size of specimen. Such increment in fractal dimension is due to the widening of FPZ. Size effect on b-value may appear insignificant, if absolute b-values are considered, due to small difference between b-values and the reason behind such insignificance

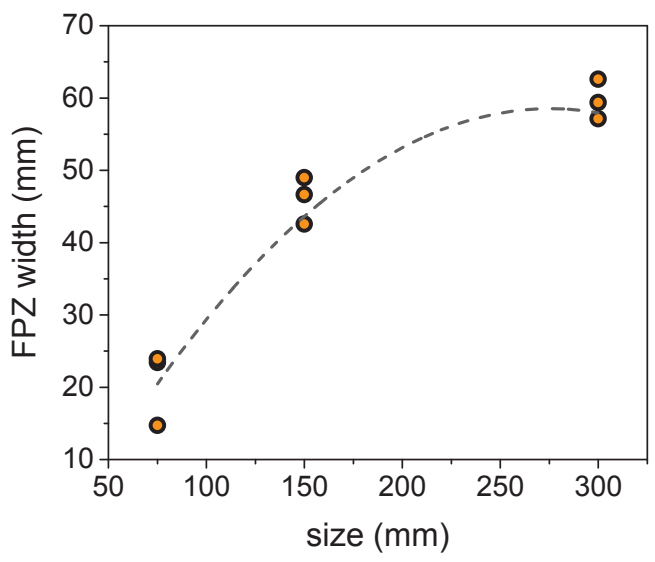

Fig. 10. Width of FPZ vs. size of the specimen. 


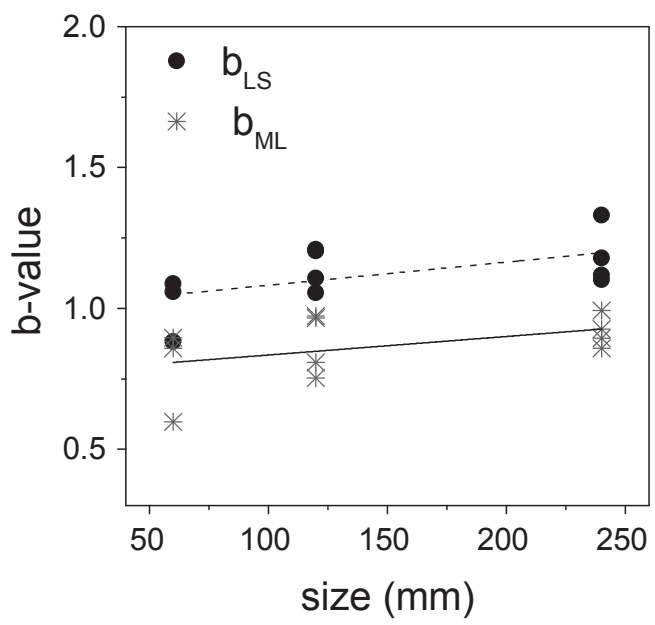

Fig. 11. Effect of finite size on b-value near failure.

is the low variability range of b-value itself. The range of b-value for concrete has been reported in the range $0.5-1.5$. Though $b$-value variation for different sizes appears insignificant, the trend is considerable and needs further detailed investigation.

\subsection{Application of GLE for b-value analysis}

The applicability of GLE for b-value analysis is demonstrated in two steps in this section. First, its superiority of fitting the CFD compared to GR law is demonstrated by fitting 1000 randomly sampled AE events from a tested beam. Then it is applied to the whole population of events up to failure in chronological order to observe time evolution of damage. The parameter s-value obtained from GLE fit is compared with GR b-value and their equivalent behavior is illustrated. The b-values obtained from GR and GLE are not the same, in fact it is argued that the $b_{G L E}$ is more generalized and informative than $b_{G R}$.

\subsubsection{Application to randomly sampled 1000 events}

Before proceeding to the application of GLE to tested beams, this section demonstrates the comparative quality of fitting by GLE and GR. To testify, 1000 randomly sampled events are taken from the population of events acquired from a tested beam up to failure. The samples are not consecutive in time rather sampled randomly to eliminate any bias. To determine $b_{G L E}$, Eq. (2) is fitted to the CFD (shown in Fig. 6a) of these sampled events. For $C=1000$, the parameters s-value and $\alpha$ are obtained and substituted into Eq. (5) to obtain $b_{G L E}$. Fig. 12 shows a comparative fit of GR and GLE. One can observe that the GR law does not fit for the small magnitudes on a linear scale although it shows the apparent fit only on the log-linear scale. The residual errors for large magnitudes appear large while for small magnitudes appear small on the log-linear scale. Therefore, the least square regression on the log-linear scale provides apparent fit for the GR law and biased to large magnitudes. On the other hand, GLE does not need the use of log-linear scale and fits CFD on the linear scale itself without showing any magnitude bias. The superiority of GLE fit is clearly seen on both linear and loglinear scale. It is important to stress that the GLE uses two parameters for fitting CFD and perhaps it is an obvious reason for a better

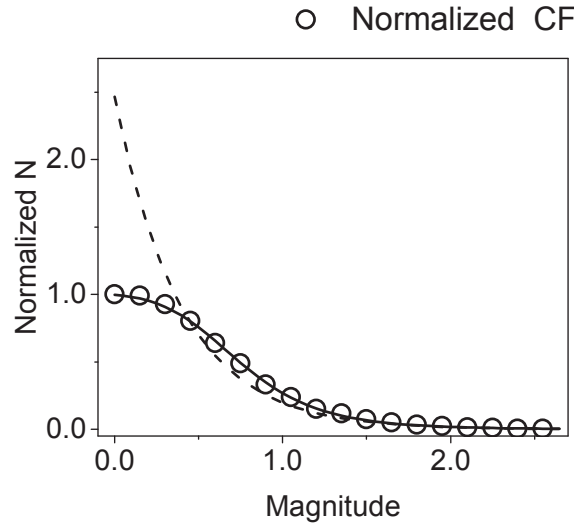

(a)

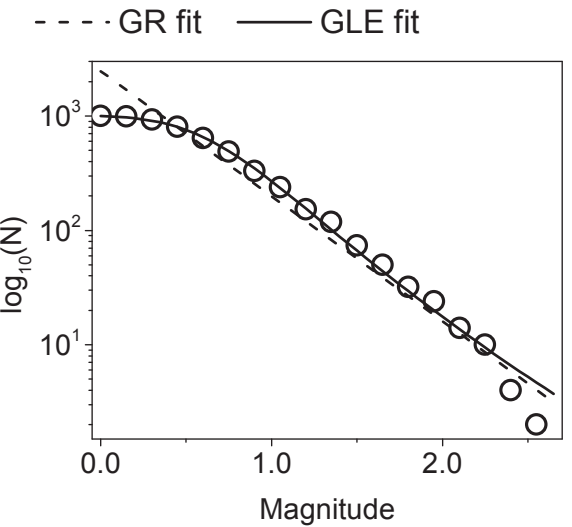

(b)

Fig. 12. GR vs GLE fit for $1000 \mathrm{AE}$ events on (a) linear scale and (b) log-linear scale. 
fit whereas GR law uses a single rate parameter.

Despite the simplicity of GR law, if it does not fit small magnitude events with considerable accuracy then the use of GR law should be avoided at least for AE in quasi-brittle materials as far as damage assessment is concerned. Use of GLE is justifiable due to its superior quality of fitting CFD for small as well as for large magnitude events. Accounting small magnitude events is necessary as they represent microcracking. Microcracking is a benefit offered by quasi-brittle material for reducing crack severity and it should not be overlooked. Hence, microcracking is an essential part of the FPZ development and neglecting microcrack contribution to b-value can result in partial information regarding damage progress.

\subsubsection{Application to AE events acquired up to failure of a beam}

As superiority of GLE over GR law is demonstrated in the previous section, its application for damage analysis of beams is explored further. Eq. (2) is used to fit CFD of AE events at various time instances during the progress of damage in the beams. The constant $C$ is taken as $C=N(0)$ at each time instant and other parameters of GLE are determined with coefficient of determination not less than 0.99. The time of 100th event is indicated after which the b-value analysis is performed to avoid sample size bias. Before illustrating the progress of $b_{G L E}$ with damage, it is helpful to understand the analogues behavior of one of its parameter, the s-value with $b_{G R}$. Fig. 13 shows the progress of s-value along with $b_{L S}$ and $b_{M L}$. The overall trend of s-value correlates with GR b-values indicating that the s-value is negatively correlated to stress (as GR b-value is negatively correlated to stress). Consequently, the svalue can be considered as a representative of the CFD slope although not being its physical slope (slope of a curve varies at every point and thus the s-value can be considered as a slope of CFD curve in an average sense). Accordingly, the GR b-value and GLE svalue are equivalent but not equal quantities. Fig. 14 plotted over the test duration shows evolution of $b_{G L E}$ along with s-value and $\alpha$. As damage progresses, $\alpha$ increases approximately from 0.35 to 0.7 indicating shift from small to large magnitude event towards failure. Fluctuations in $\alpha$ and s-value show the sensitivity of these parameters for changes in CFD and such fluctuations are averaged out in $b_{G L E}$.

Fig. 15 shows the evolution of $b_{G L E}$ along with applied load on a normalized time axis for three different sizes of beam specimens. The growth of $b_{G L E}$ starts approximately at 70-80\% of peak load in prepeak regime where 100th event is detected. The initial growth rate of $b_{G L E}$ is steep due to accelerated damage growth. As crack grows and load drops due to softening behavior, the damage growth stabilizes due to various toughening mechanism taking place as a result of microcracking and $b_{G L E}$ approaches a constant value near failure. It is important to notice that the $b_{G L E}$ increases monotonically (with some fluctuations) up to failure. This behavior of $b_{G L E}$ is consistent with damage growth and hence it can be considered as a damage compliant b-value.

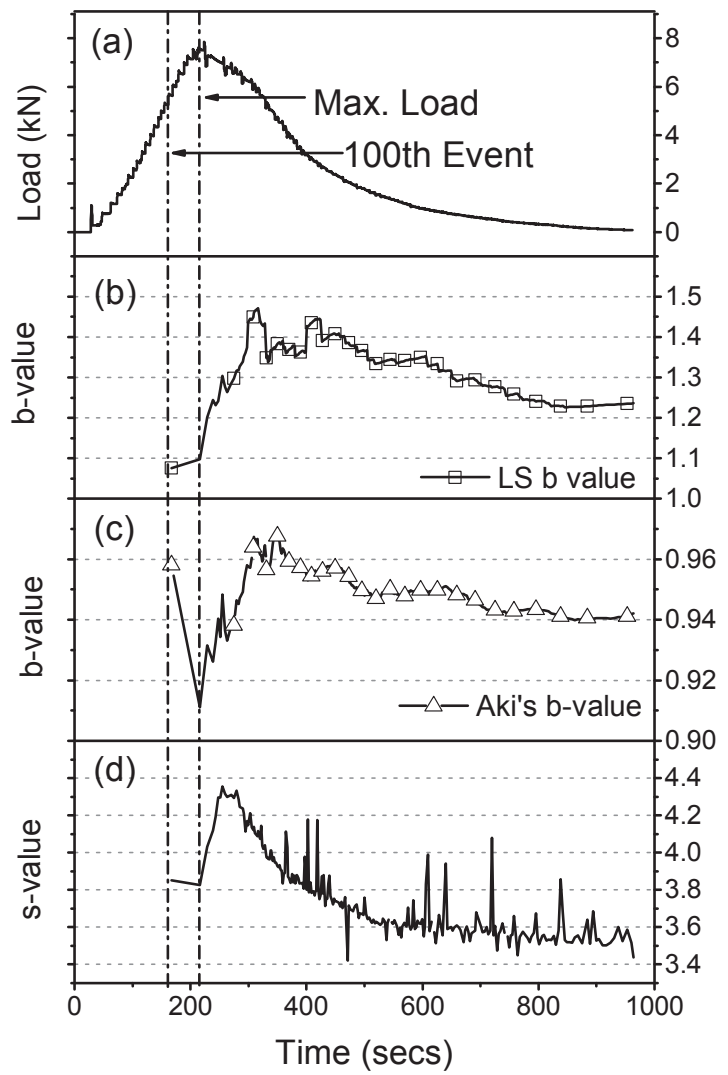

Fig. 13. (a) Load vs. time, comparison between (b) least square b-value, (c) Aki's b-value and (d) s-value of GLE. 


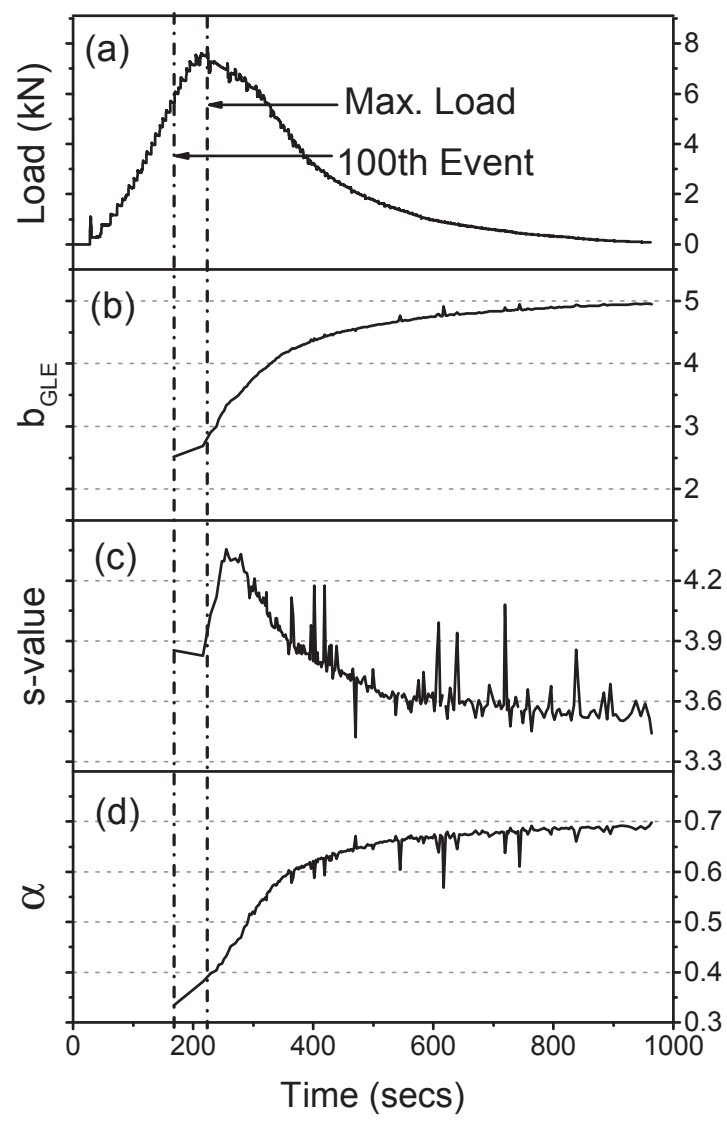

Fig. 14. (a) Load vs time, (b) $b_{G L E}$, (c) s-value, (d) $\alpha$.

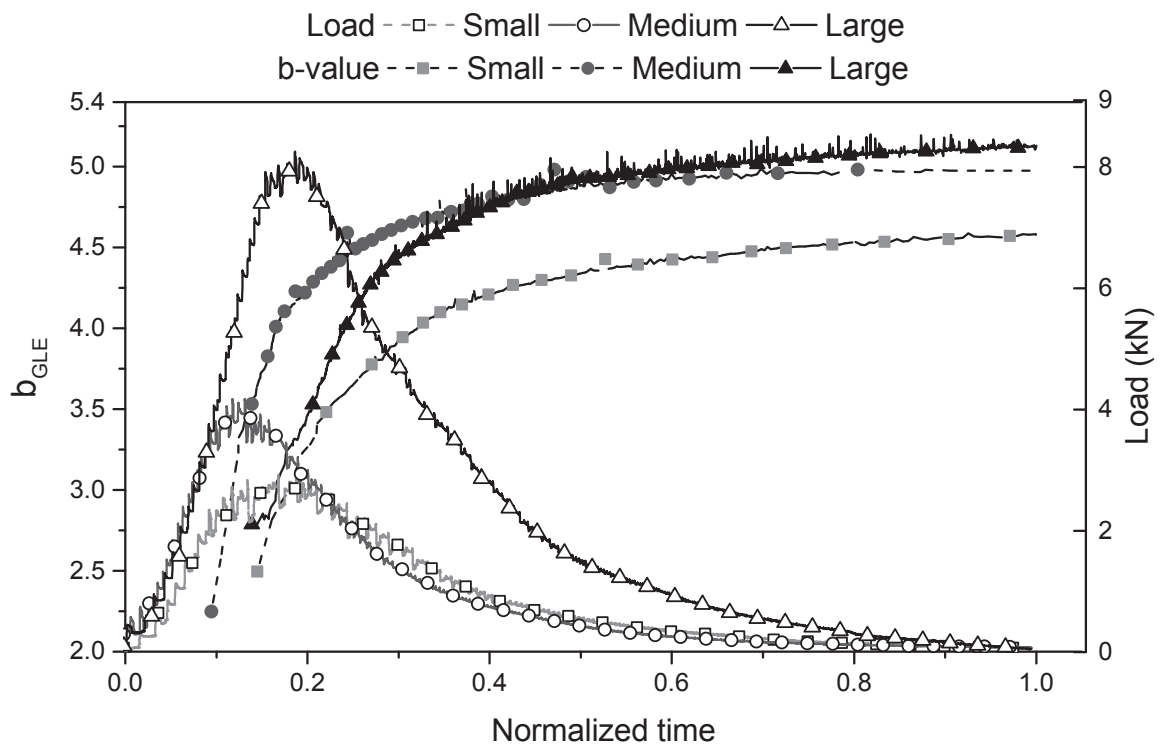

Fig. 15. Typical evolution of $b_{G L E}$ for three different size specimen.

\section{4. $b_{G L E}$ versus effective crack length}

To determine a one to one relation between crack size and event magnitude is infeasible. Therefore, the causal inference is inevitable for deriving some important conclusions in the present work. The GR b-value and GLE s-value have been shown correlated 
negatively to stress. Therefore, an important question that needs to be answered here is, if the stress causes variation in s-value, then what is the source of incremental cut-off magnitude in CFD? The obvious explanation comes from the mechanics of fracture of the problem. The far-field applied stress may cause crack propagation but the state of stress near the crack tip is defined by the stress intensity factor (SIF). The crack interaction of different sizes (as discussed in Section 7.1) relieves stress singularity and restricts crack growth which results in cut-off magnitude. The parameter $\alpha$ accounts for nonlinearity and cut-off magnitude of CFD and therefore it must be correlated to the SIF at the instance of the damage. Considering the general expression of stress intensity factor for mode-I as,

$$
K_{I}=\sigma \sqrt{\pi a} f(\bar{a}) \Rightarrow a=\frac{1}{\pi f(\bar{a})}\left(\frac{K_{I}}{\sigma}\right)^{2}
$$

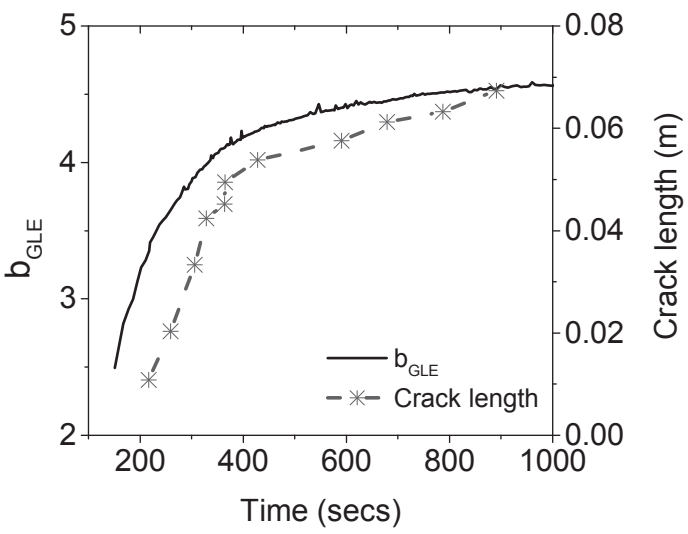

(a)

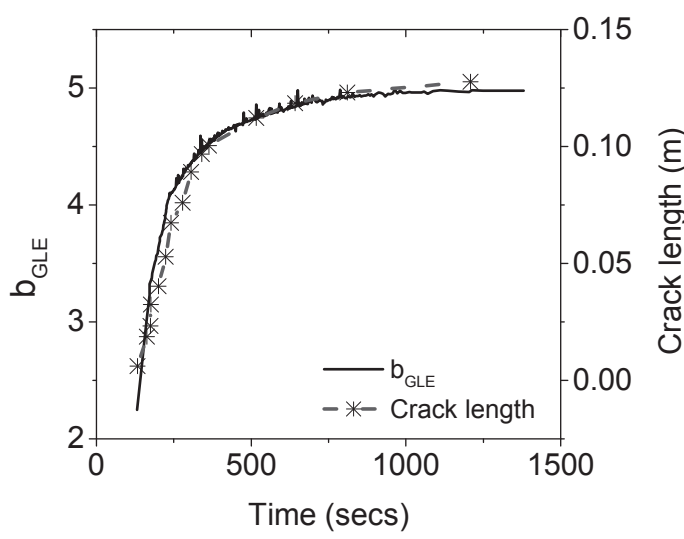

(b)

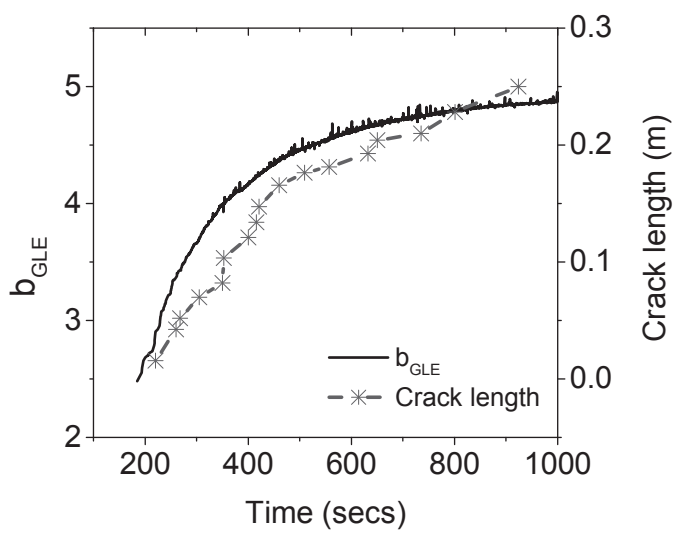

(c)

Fig. 16. Correlation between crack length and b-value of GLE for (a) small, (b) medium and (c) large size specimen. 
where $K_{I}$ is the mode-I stress intensity factor due to stress $\sigma$ at crack length $a . f(\bar{a})$ is geometry shape factor and $\bar{a}=a / d$, where $d$ is size of the specimen. Eq. (6) is applicable to brittle materials and does not account for different crack sizes and their interaction but for simplicity of argument this equation serves the purpose. According to linear elastic fracture mechanics, Eq. (6) implies that the effective crack length at any point during damage progression can be expressed as a ratio of stress intensity factor and stress normalized by geometry shape function as follows,

$$
a_{e} \approx f\left(\sigma, K_{I}, \text { geometry }\right)
$$

The parameter $\alpha$ increases with nonlinearity and cut-off magnitude in CFD and therefore it can be considered as correlated to SIF as $K_{I} \propto \alpha \propto \frac{1}{(1-\alpha)}$ and the stress can be correlated to s-value as $s \propto 1 / \sigma$. Therefore, for the same geometry,

$$
b_{G L E}=\frac{s}{(1-\alpha)} \Rightarrow f\left(\sigma, K_{I}\right)
$$

Eq. (8) signifies that the $b_{G L E}$ is a function of stress and stress intensity factor in distribution domain. Consequently, a change in $b_{G L E}$ can be considered correlated to effective crack length during damage progress.

$$
\frac{\Delta b_{G L E}}{\Delta t} \propto \frac{\Delta a_{e}}{\Delta t}
$$

Fig. 16 shows $b_{G L E}$ on the left and effective crack length on the right axis plotted over time to demonstrate the correlation expressed by Eq. (9). The effective crack is determined from digital image correlation technique as discussed in Sections 5.2 and 6 . A reasonably good correlation as seen between effective crack length and $b_{G L E}$ illustrates the damage compliant behavior of $b_{G L E}$.

\section{5. $b_{G L E}$ versus scale effect}

Fig. 17 shows the effect of finite specimen size on $\alpha$ and s-value which eventually results in scale effect on $b_{G L E}$. Data scatter is noticeable in small size beams and it is negligible for large beams. The reason behind scatter of $\alpha$ and s-value in small beams is obviously due to prominent heterogeneity effect (heterogeneity of material is unchanged but its effect gets intense with decrease in dimensions of the beams). As beam size increases, such heterogeneity effect averages out resulting in reduced scatter in parameters. Similar to b-values determined by GR law as shown in Fig. 11, the size effect on $b_{G L E}$ also appears negligible but the trend is again significant. The observed ranges of GLE parameters are shown in Table 3.

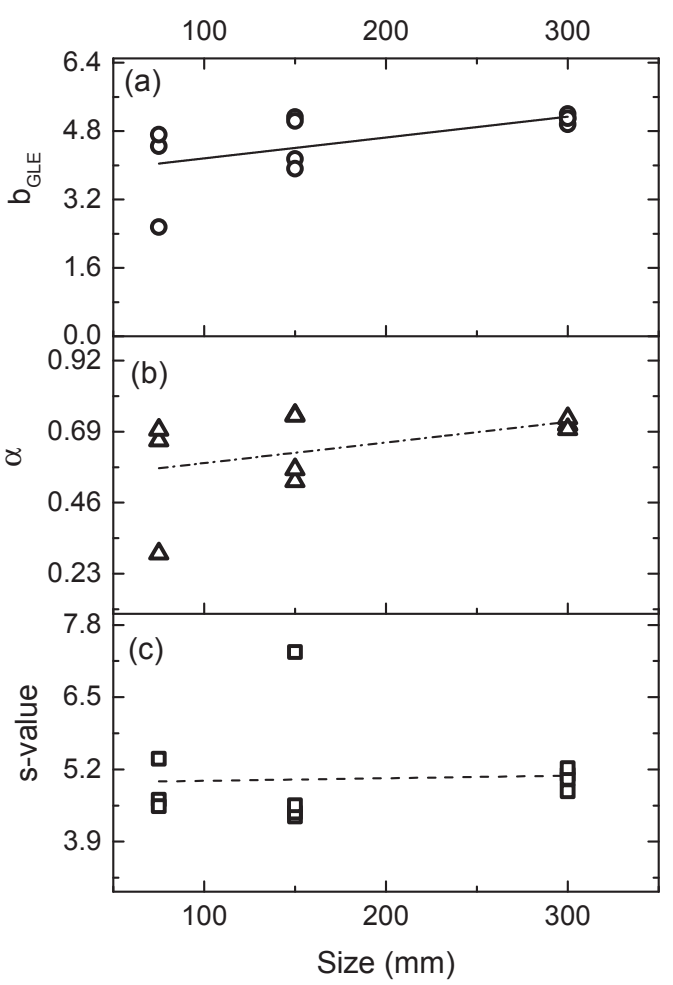

Fig. 17. Effect of finite size on b-value obtained from GLE. 
Table 3

Range of different parameters.

\begin{tabular}{ll}
\hline Parameter & Range \\
\hline s-value & $4.0-7.5$ \\
$\alpha$ & $0.3-0.75$ \\
$b_{G L E}$ & $2.5-5.5$ \\
\hline
\end{tabular}

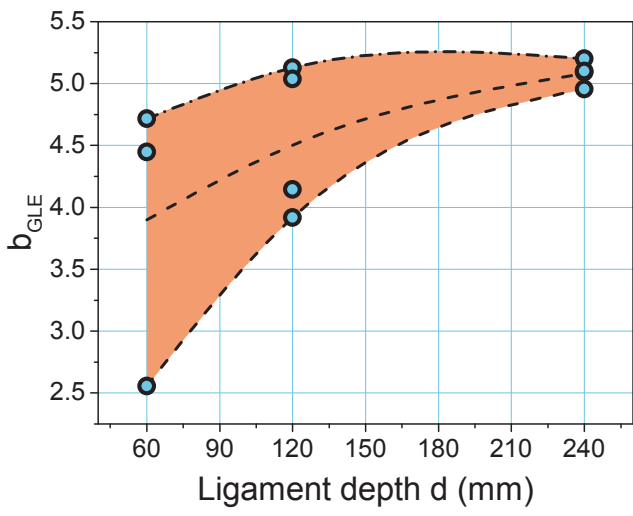

(a)

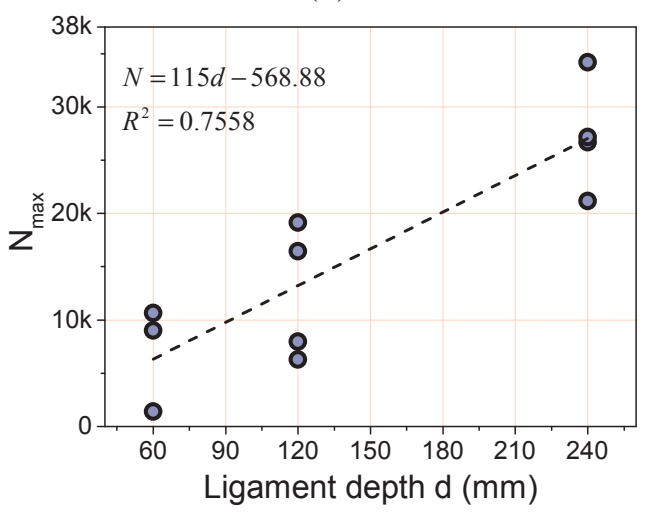

(c)

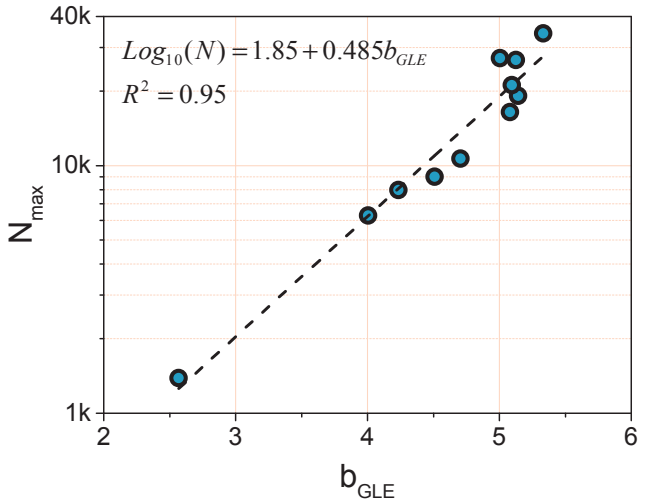

(e)

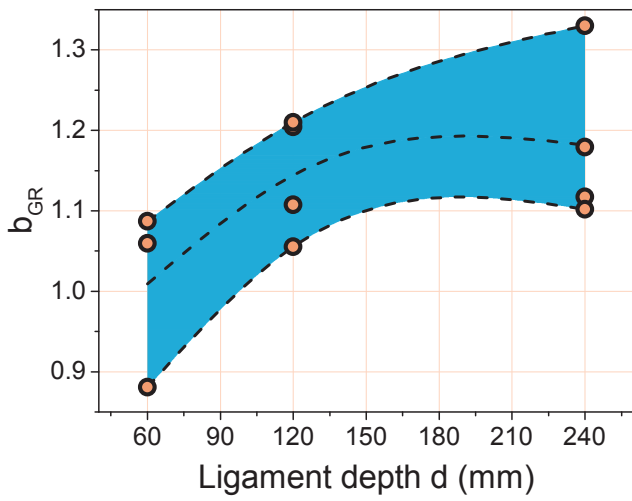

(b)

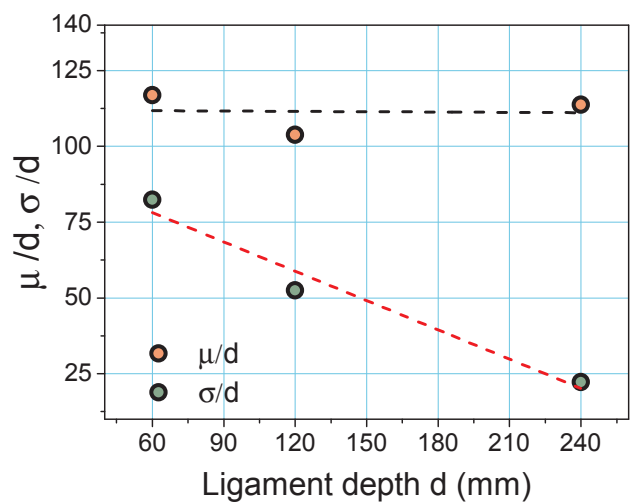

(d)

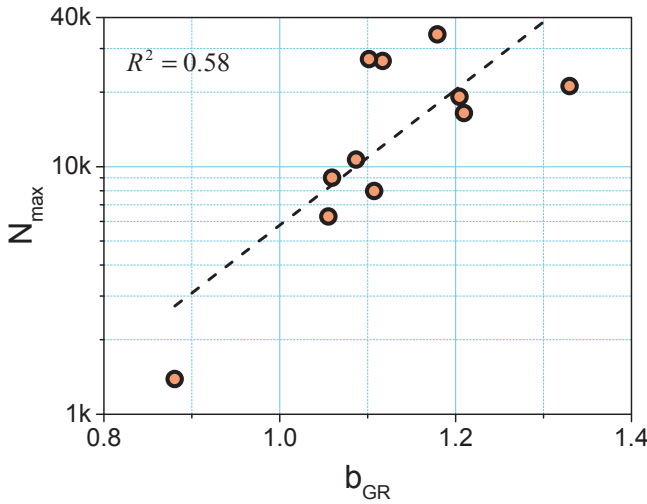

(f)

Fig. 18. Envelope of (a) $b_{G L E}$ and (b) $b_{G R}$ for three ligament depths, (c) linear fit between $N_{\max }$ and ligament depth, (d) normalizes mean ( $\mu$ ) and standard deviation $(\sigma)$ of $N_{\max }$, log-linear relationship between $N_{\max }$ and (e) $b_{G L E}$ and (f) $b_{G R}$. 


\subsection{Uncertainty of $b_{G L E}$ versus $b_{G R}$}

The b-values obtained from GLE and GR law show different scatter pattern with respect to ligament depth of the beams as shown in Fig. 18a and b enveloped by maximum and minimum values with average nonlinear trend (dashed lines). The uncertainty in bvalue is a result of uncertain occurrence of cracks in concrete due to its heterogeneous microstructure which produces uncertain events of varying magnitudes. To compare uncertain behavior of the material at different scales, the AE information needs to be normalized by volume of FPZ to obtain crack density. However the volume of FPZ is unknown and therefore the ligament depth is used as a representative of beam size for normalization. The mean $(\mu)$ of $N_{\max }$ normalized by ligament depth measures mean events per unit ligament depth. Similarly, normalized standard deviation of events for a beam size can be determined. Fig. 18d shows the variation of normalized mean and standard deviation $(\sigma)$ of $N_{\max }$ derived from statistics of AE events listed in Table 2. Though cumulative number of events increases with increase in beam size as shown in Fig. 18c, the normalized dispersion of these events reduces with the increase in beam size as shown in Fig. 18d. This illustrates that the uncertainty of AE generation in concrete reduces with larger beam size. Here, uncertainty of AE detectability is not considered as AE sensors are arranged closely around the crack path to avoid any data loss by attenuation.

The observed scatter of $b_{G L E}$ (Fig. 18a) reflects this uncertainty caused by beam size whereas dispersion of $b_{G L E}$ reduces for large beam sizes. On the contrary, $b_{G R}$ does not account for AE generation uncertainty. Fig. 18e shows a linear relation between $N_{m a x}$ and $b_{G L E}$ on a log-linear scale. As $N_{\max }$ can be predicted by relating it with ligament depth of beam, existence of relationship between $N_{\max }$ and $b_{G L E}$ should help to predict expected $b_{G L E}$ for damage evaluation. Such type of relation is not certain for $b_{G R}$ as shown in Fig. $18 \mathrm{f}$.

\section{Conclusions}

The GR distribution has been successfully used for effective prediction of final failure in structural elements. Despite such productivity of $b_{G R}$, its bias for certain magnitude range and inadequacy to characterize CFD makes it uncertain for quantitative damage evaluation. Currently, two critical stages, one near peak load and other near failure, are used to characterize damage where b-value varies from 1.5 to 1.0 respectively. The present work emphasis on monotonic increase in b-value with incremental damage such that the damage can be characterized in between these two critical conditions quantitatively. If one looks closer to CFD evolving over time, as an animated series of curves superposed in chronological order, the subtle changes in its shape become visible with progressive damage. Quantification of these shape changes by single parameter b-value oversimplifies and approximates the nonlinearity in CFD. One to one correlation between damage level and $b_{G R}$ is also absent as different damage levels sometimes show the same bvalues. On the other hand, the logistic (sigmoidal) growth of microcracks is more appealing, natural and evident in experimental studies over the power law growth. The two parameters, s-value as a rate parameter and $\alpha$ as a shape parameter of GLE, effectively captures the subtle shape changes of CFD. The proposed $b_{G L E}$ is rather a generalized version of $b_{G R}$ which not only fits well in the whole range of magnitude but accounts for cut-off magnitude too. GLE approaches the problem in more statistical way as a problem of parameter estimation without the need of log-linear scale transformation whereas GR law is merely a linear least square fitting technique on log-linear scale. Moreover, the monotonic incremental behavior of $b_{G L E}$ makes it damage compliant as it correlates with crack length. Therefore, one to one correlation between damage level and $b_{G L E}$ is possible to evaluate damage quantitatively. Accommodation of AE generation uncertainty and size effect makes $b_{G L E}$ superior and predictable over $b_{G R}$ for practical applications.

In practice, damage initializes by dispersed micro cracking and then a dominant crack takes over the damage process. In such cases $b_{G L E}$ can be effectively used to evaluate damage quantitatively. Small size geometrically similar specimens are often tested in laboratories to understand the behavior of real world large structural elements. The fracture mechanics based research on concrete revolves around quantifying the prominently observed size effect through such laboratory experiments. Therefore, it is also necessary to evaluate size effect on b-value for its consideration as an absolute damage indicator for structural health monitoring. Therefore, the present work is motivated by the fact that instead of apparent universality, an absolute b-value near failure for each size of beam can be determined in laboratory and can be extrapolated to account for size effect in real world application.

\section{References}

[1] Gutenberg G, Richter C. Seismicity of the earth and associated phenomena. J Geophys Res 1950;55:97.

[2] Carpinteri A, Lacidogna G, Pugno N. Richter's laws at the laboratory scale interpreted by acoustic emission. Mag Concr Res 2006;58(9):619-26.

[3] Aki K. Maximum likelihood estimate of b in the formula $\log \mathrm{N}=\mathrm{a}$-bm and its confidence limits. Bull Earthq Res Inst, Tokyo Univ 1965;43:237-9.

[4] Shiotani T. Evaluation of progressive failure using AE sources and improved b-value on slope model tests. Prog Acoust Emission VII, JSNDI 1994:529-34.

[5] Weichert DH. Estimation of the earthquake recurrence parameters for unequal observation periods for different magnitudes. Bull Seismol Soc Am 1980;70(4):1337-46.

[6] Kijko A, Smit A. Extension of the Aki-Utsu b-value estimator for incomplete catalogs. Bull Seismol Soc Am 2012;102(3):1283-7.

[7] Han Q, Wang L, Xu J, Carpinteri A, Lacidogna G. A robust method to estimate the b-value of the magnitude-frequency distribution of earthquakes. Chaos, Solitons Fract 2015;81:103-10.

[8] Abe S, Okamoto Y. Nonextensive statistical mechanics and its applications vol. 560. Springer Science \& Business Media; 2001.

[9] Sotolongo-Costa O, Posadas A. Fragment-asperity interaction model for earthquakes. Phys Rev Lett 2004;92(4):048501.

[10] Silva R, França G, Vilar C, Alcaniz J. Nonextensive models for earthquakes. Phys Rev E 2006;73(2):026102.

[11] Sánchez CE, Pedro V-J. New Bayesian frequency-magnitude distribution model for earthquakes applied in Chile. Physica A: Stat Mech Appl 2018;508:305-12.

[12] Maslov LA, Chebotarev VI. Modeling statistics and kinetics of the natural aggregation structures and processes with the solution of generalized logistic equation. Physica A: Stat Mech Appl 2017;468:691-7.

[13] Carpinteri A, Lacidogna G. Earthquakes and acoustic emission: selected papers from the 11th international conference on fracture, Turin, Italy, March 20-25, 2005. CRC Press; 2007.

[14] Cosentino P. Difficulties and related criticism in applying the Gutenberg and Richter relation to the seismic regions in statistical seismology. Boll Geof Teor Appl 
1976;70:79-91.

[15] Carpinteri A, Lacidogna G, Accornero F, Mpalaskas A, Matikas T, Aggelis D. Influence of damage in the acoustic emission parameters. Cem Concr Compos 2013;44:9-16.

[16] Carpinteri A, Lacidogna G, Pugno N. Structural damage diagnosis and life-time assessment by acoustic emission monitoring. Eng Fract Mech 2007;74(1-2):273-89.

[17] Shah SG, Chandra Kishen JM. Fracture behavior of concrete-concrete interface using acoustic emission technique. Eng Fract Mech 2010;77(6):908-24.

[18] Muralidhara S, Raghu Prasad BK, Eskandari H, Karihaloo BL. Fracture process zone size and true fracture energy of concrete using acoustic emission. Construct Build Mater 2010;24(4):479-86.

[19] Lacidogna G, Piana G, Carpinteri A. Acoustic emission and modal frequency variation in concrete specimens under four-point bending. Appl Sci 2017;7(4):339.

[20] Colombo IS, Main I, Forde M. Assessing damage of reinforced concrete beam using "b-value" analysis of acoustic emission signals. J Mater Civ Eng 2003;15(3):280-6.

[21] Pacheco JF, Scholz CH, Sykes LR. Changes in frequency-size relationship from small to large earthquakes. Nature 1992;355(6355):71.

[22] Knopoff L. The magnitude distribution of declustered earthquakes in southern california. Proc Natl Acad Sci 2000;97(22):11880-4.

[23] Kagan Y. Universality of the seismic moment-frequency relation. Seismicity patterns, their statistical significance and physical meaning. Springer; 1999. p. $537-73$.

[24] Carpinteri A, Lacidogna G, Puzzi S. From criticality to final collapse: evolution of the "b-value" from 1.5 to 1.0. Chaos, Solitons Fract 2009;41(2):843-53.

[25] Amitrano D. Variability in the power-law distributions of rupture events. Eur Phys J Special Top 2012;205(1):199-215.

[26] Mogi K. Magnitude-frequency relation for elastic shocks accompanying fractures of various materials and some related problems in earthquakes. Bull Earthq Res Inst, Univ Tokyo 1962;40:831-53.

[27] Mihashi H, Nomura N. Correlation between characteristics of fracture process zone and tension-softening properties of concrete. Nucl Eng Des 1996;165(3):359-76.

[28] Scholz C. The frequency-magnitude relation of microfracturing in rock and its relation to earthquakes. Bull Seismol Soc Am 1968;58(1):399-415.

[29] Sammonds PR, Meredith P, Murrell S, Main I, et al. Modelling the damage evolution in rock containing pore fluid by acoustic emission. Rock mechanics in petroleum engineering. Society of Petroleum Engineers; 1994.

[30] Lockner D. The role of acoustic emission in the study of rock fracture. International journal of rock mechanics and mining sciences \& geomechanics abstracts, vol. 30. Elsevier; 1993. p. 883-99.

[31] Amitrano D, Grasso JR, Senfaute G. Seismic precursory patterns before a cliff collapse and critical point phenomena. Geophys Res Lett 2005;32(8).

[32] Main I. Apparent breaks in scaling in the earthquake cumulative frequency-magnitude distribution: fact or artifact? Bull Seismol Soc Am 2000;90(1):86-97.

[33] Marzocchi W, Sandri L. A review and new insights on the estimation of the b-value and its uncertainty. Ann Geophys 2003;46:1271-82.

[34] Utsu T. A method for determinating the value of b in a formula $\log \mathrm{N}=\mathrm{a}$-bM showing the magnitude-frequency relation for earthquakes. Geophys Bill Hokkaido Univ 1965;13:99-103.

[35] Tinti S, Mulargia F. Confidence intervals of b values for grouped magnitudes. Bull Seismol Soc Am 1987;77(6):2125-34.

[36] Clauset A, Shalizi CR, Newman ME. Power-law distributions in empirical data. SIAM Rev 2009;51(4):661-703.

[37] Kagan Y. Seismic moment-frequency relation for shallow earthquakes: regional comparison. J Geophys Res: Solid Earth 1997;102(B2):2835-52.

[38] Kagan YY. Seismic moment distribution revisited: I. Statistical results. Geophys J Int 2002;148(3):520-41.

[39] Brencich A. The microcrack-interacting model. Nonlinear crack models for nonmetallic materials. Springer; 1999. p. 209-84.

[40] Otsuka K, Date H. Fracture process zone in concrete tension specimen. Eng Fract Mech 2000;65(2-3):111-31.

[41] Ohno K, Uji K, Ueno A, Ohtsu M. Fracture process zone in notched concrete beam under three-point bending by acoustic emission. Construct Build Mater 2014;67:139-45.

[42] Gom correlate, gom, braunschweig, Germany; 2017. [online] < https://www.gom.com/3d-software/gom-correlate.html >

[43] Sornette D, Sornette A. General theory of the modified Gutenberg-Richter law for large seismic moments. Bull Seismol Soc Am 1999;89(4):1121-30.

[44] Carpinteri A. Scaling laws and renormalization groups for strength and toughness of disordered materials. Int J Solids Struct 1994;31(3):291-302.

[45] Aki K. A probabilistic synthesis of precursory phenomena. Earthq Predict: Int Rev 1981;4:566-74. 